\title{
Maternal diet disrupts the placenta-brain axis in a sex-specific manner
}

Alexis M Ceasrine ${ }^{1}$, Benjamin A Devlin ${ }^{1}$, Jessica L. Bolton², Young Chan Jo1, Carolyn Huynh ${ }^{1}$, Bailey Patrick ${ }^{1}$, Kamryn Washington ${ }^{1}$, Faith Joo ${ }^{1}$, A. Brayan Campos-Salazar ${ }^{3}$, Elana R. Lockshin ${ }^{3}$, Susan K. Murphy ${ }^{4}$, Leigh Ann Simmons ${ }^{5}$, Staci D. Bilbo*1,3,6

${ }^{1}$ Department of Psychology and Neuroscience, Duke University, Durham, NC, USA

${ }^{2}$ Neuroscience Institute, Georgia State University, Atlanta, GA, USA

${ }^{3}$ Department of Neurobiology, Duke University Medical Center, Durham, NC, USA

${ }^{4}$ Obstetrics and Gynecology Duke University Medical Center, Durham, NC, USA

${ }^{5}$ Department of Human Ecology, Perinatal Origins of Disparities Center, University of California, Davis, California, USA

${ }^{6}$ Lurie Center for Autism, Massachusetts General Hospital, Boston, MA

SUMMARY: High maternal weight is associated with a number of detrimental outcomes

in offspring, including increased susceptibility to neurological disorders such as anxiety, depression, and communicative disorders (e.g. autism spectrum disorders) ${ }^{1-4}$. Despite widespread acknowledgement of sex-biases in the prevalence, incidence, and age of onset of these disorders, few studies have investigated potential sex-biased mechanisms underlying disorder susceptibility. Here, we use a mouse model to demonstrate how maternal high-fat diet causes perinatal inflammation that influences sex-specific behavioral outcomes in offspring. In male high-fat diet offspring, increased macrophage toll like receptor 4 signaling results in excess phagocytosis of serotonin neurons in the developing dorsal raphe nucleus, decreasing serotonin bioavailability in the fetal and adult brain. Bulk sequencing from a large cohort of matched first trimester human fetal brain, placenta, and maternal decidua samples reveals sex-specific transcriptome-wide changes in placenta and brain tissue. Further, we find that fetal brain serotonin is significantly negatively correlated with maternal triglyceride accumulation (a proxy for dietary fat content) in male pregnancies only. These findings uncover a fundamental mechanism through which maternal diet may increase offspring susceptibility for neuropsychiatric disorder development. 
MAIN: In the United States, more than $50 \%$ of women have overweight or obesity when they become pregnant ${ }^{5}$. Decades of research into anxiety and depression have strongly implicated disruptions in the serotonin system as causal to disease, though treatment efficacy (via selective serotonin reuptake inhibitors) is low, and little has been done to investigate this on the basis of sex. Moreover, despite being female-biased $(1: 1.7-2)^{6,7}$, a significant number of adult men are affected by these conditions (3-6\% of men affected by major depression; up to $10 \%$ by generalized anxiety $)^{8}$. Understanding the developmental mechanisms through which sex contributes to neurological disorder development, susceptibility, and severity is of utmost importance when considering clinical implications such as treatment efficacy.

\section{Maternal high-fat diet imparts sex-specific offspring behavioral outcomes}

To model high maternal weight in mice, female wild-type (C57BL/6J) mice were placed on either a high-fat ( $45 \% \mathrm{kcal}$ from fat; HFD) or low-fat ( $10 \% \mathrm{kcal}$ from fat, sucrose matched; LFD) diet for six weeks prior to mating (Fig 1a). HFD females gained significantly more weight than LFD females before pregnancy and their weight remained elevated throughout gestation (Extended Data Fig 1a-b). No significant differences were observed in litter size, composition, or maternal care (during either the light or dark phase; Extended Data Fig 1c-e). Both male and female maternal high-fat diet (mHFD) offspring weighed significantly more than maternal low-fat diet (mLFD) offspring (Extended Data Fig 1f). Offspring behavior was assessed at neonatal, juvenile, and adult time-points (Fig 1a). Behaviors are presented sex-stratified as they are often sex-dependent in their presentation $^{9,10}$. 
Fig 1. Maternal high-fat diet imparts sex-specific offspring behavioral outcomes.
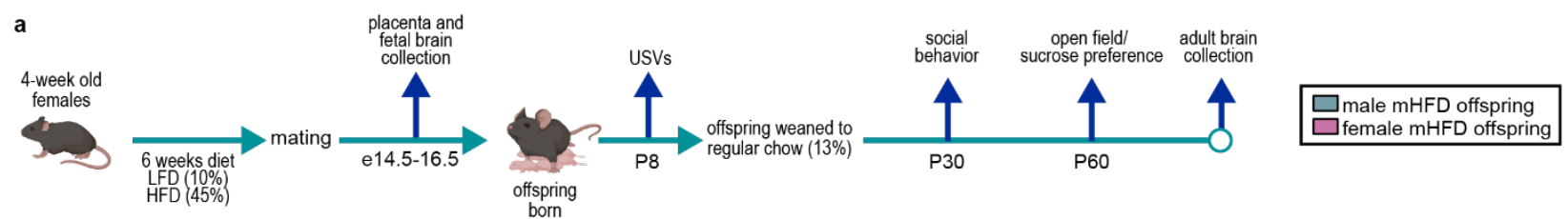

b
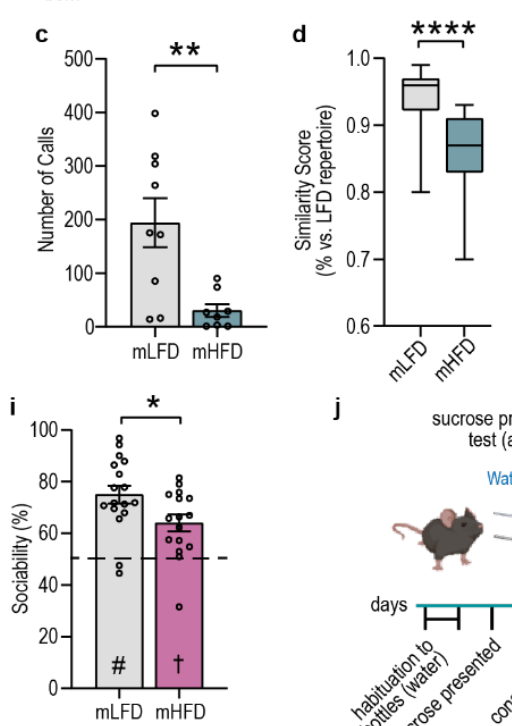

j

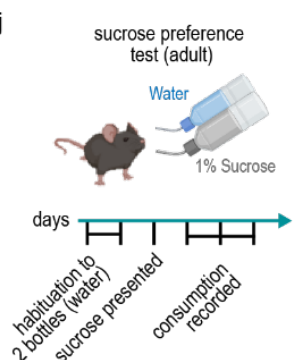

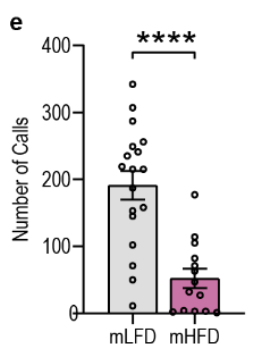
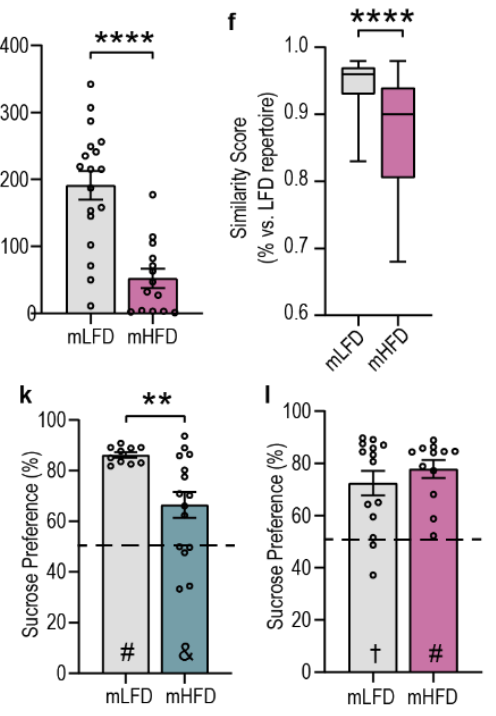

a, Schematic of maternal low-fat and high-fat diet paradigm ( $10 \%$ and $45 \%$ represent $\%$ kcal from fat in diet). b, Schematic of neonatal maternal separation-induced ultrasonic vocalization (USV) recording and analysis. c-f, mHFD decreases neonatal USV number and syllable repertoire similarity in male and female offspring ( $n=9$ mLFD, 8 mHFD male offspring; 18 mLFD, 14 mHFD female offspring). g, Schematic of 3-chambered social preference test. h, Male mLFD and mHFD offspring display a strong social preference ( $n=13 \mathrm{mLFD}, 15 \mathrm{mHFD}$ male offspring). i, Female mHFD offspring have a reduced social preference versus mLFD offspring ( $n=17 \mathrm{mLFD}, 16 \mathrm{mHFD}$ female offspring). j, Schematic of sucrose preference test. $\mathbf{k}$, Male mHFD offspring display a decreased preference for sucrose versus $\mathrm{mLFD}$ offspring ( $\mathrm{n}=10 \mathrm{mLFD}, 15 \mathrm{mHFD}$ male offspring). I, Female $\mathrm{mLFD}$ and $\mathrm{mHFD}$ offspring display a strong sucrose preference ( $n=14 \mathrm{mLFD}, 12 \mathrm{mHFD}$ female offspring). Data are mean \pm s.e.m.; $n$ represents biologically independent mice. asterisk denoted $p$-values are derived from unpaired two-tailed t-tests $(\mathbf{c}, \mathbf{e}, \mathbf{i}, \mathbf{k})$ or paired two-tailed t-tests $(\mathbf{d}, \mathbf{f})$. other $p$-values $(\# \mathbf{p}<0.0001, \dagger p<0.001, \&$ $\mathrm{p}<0.01)$ are derived from one-sample t-tests assessing difference from chance $(50 \%)(\mathbf{h}, \mathbf{i}, \mathbf{k}, \mathbf{I})$.

Neonatal maternal separation-induced ultrasonic vocalizations (USVs) provide a

readout of early communicative behaviors in mice, and peak at postnatal day 8 in C57BL/6J mice ${ }^{11,12}$. USV analyses (Fig 1b) revealed a significant decrease in the number of USVs emitted, total call time, and mean call length in both male (Fig 1c, Extended Data Fig 2a-b) and female (Fig 1e, Extended Data Fig 2c-d) mHFD offspring compared to same sex mLFD offspring. In-depth characterization of overall USV composition (integrating alterations in frequency $(\mathrm{kHz})$, energy $(\mathrm{dB})$, and syllable complexity (e.g. number of syllables, frequency of calls)) using Mouse Ultrasonic Profile ExTraction (MUPET ${ }^{13}$ ) revealed a significant decrease in USV similarity between mLFD and mHFD male (Fig 1d) and female (Fig 1f) offspring. While little is known about USV complexity in neonates, 
adult USVs are context dependent, and specific USV parameters such as frequency $(\mathrm{kHz})$ are known to vary in response to a stressful environment ${ }^{14}$. To further investigate potential behavioral deficits in mHFD offspring, we assessed juvenile sociability using a 3-chamber social preference test (Fig 1g). Male mHFD offspring showed no change in sociability or time investigating the social stimulus when compared to controls (Fig 1h; Extended Data Fig 2e). Interestingly, female mHFD offspring had a significantly decreased sociability score, driven by female mHFD offspring spending significantly less time investigating the social stimulus as compared to controls (Fig 1i; Extended Data Fig 2f). Neither male nor female mHFD offspring had any changes in general activity or anxiety-like behavior (Extended Data Fig 2i-p). This suggests that the social deficits observed in females are not due to impaired motor skills or an anxiety-like phenotype. To better understand the behavioral changes affecting male mHFD offspring, we determined average sucrose preference over a 3-day free-choice test (Fig 1j). Mice normally display a strong preference for sucrose, and diminished sucrose preference is indicative of anhedonia, or lack of reward/pleasure. Average sucrose preference was significantly decreased in male, but not female, mHFD offspring, indicative of anhedonia (Fig 1k-I). Neither male nor female mHFD offspring showed a change in total liquid consumption (water + sucrose) over the 3-day testing period (Extended Data Fig 2g-h). In sum, despite similar behavioral shifts in USVs in early life, by adolescence and into adulthood the behavior patterns shift based on sex, with mHFD female offspring showing social deficits, and mHFD male offspring showing diminished non-social reward behavior.

mHFD decreases serotonin and causes behavior changes in male offspring 
We observed altered neonatal USVs and anhedonia in male mHFD offspring. These behaviors are associated with diminished brain serotonin $(5-\mathrm{HT})$ in mice ${ }^{15,16}$. Serotonergic neurons in the brain reside in the raphe nuclei throughout the brainstem and project to many brain regions. Circuitry between the dorsal raphe nucleus (DRN) and the prefrontal cortex (PFC) is important for both social behavior and sucrose preference in rodents ${ }^{17,18}$. Thus, we first assessed serotonin levels in the PFC of adult male and female mLFD and mHFD offspring. Of note, female mLFD PFC 5-HT levels tended to be lower than male mLFD PFC 5-HT levels. While sex differences in rodent 5-HT levels have not been described for the PFC, it is well established that human males synthesize brain serotonin at significantly higher rates than females ${ }^{19}$, and male mice have been shown to have higher tissue 5-HT than females in other brain regions (e.g. hippocampus) ${ }^{20}$. In adult male mHFD offspring, PFC serotonin levels were significantly decreased compared to mLFD (Fig 2a), while we observed no changes in serotonin levels between mLFD and mHFD female offspring (Fig 2d).

Previous research has demonstrated that brain serotonin levels during discrete windows of development are dependent on placental serotonin synthesis ${ }^{21}$. The placenta is a temporary organ that develops during early gestation and surrounds the fetus to both restrict and facilitate macromolecule and nutrient transport. Further, the placenta acts as a critical interface between the maternal environment and the developing fetal brain, and responds to maternal diet/environment in a fetal sex-specific manner ${ }^{22-24}$. However, a potential role for fetal sex in placental serotonin production is unknown. Assessment of serotonin levels in mLFD and mHFD placenta and fetal forebrain revealed a male-specific decrease in tissue serotonin in mHFD offspring (Fig 2b-c, e-f). In agreement with previous 
Fig 2. mHFD decreases serotonin and causes behavior changes in male offspring
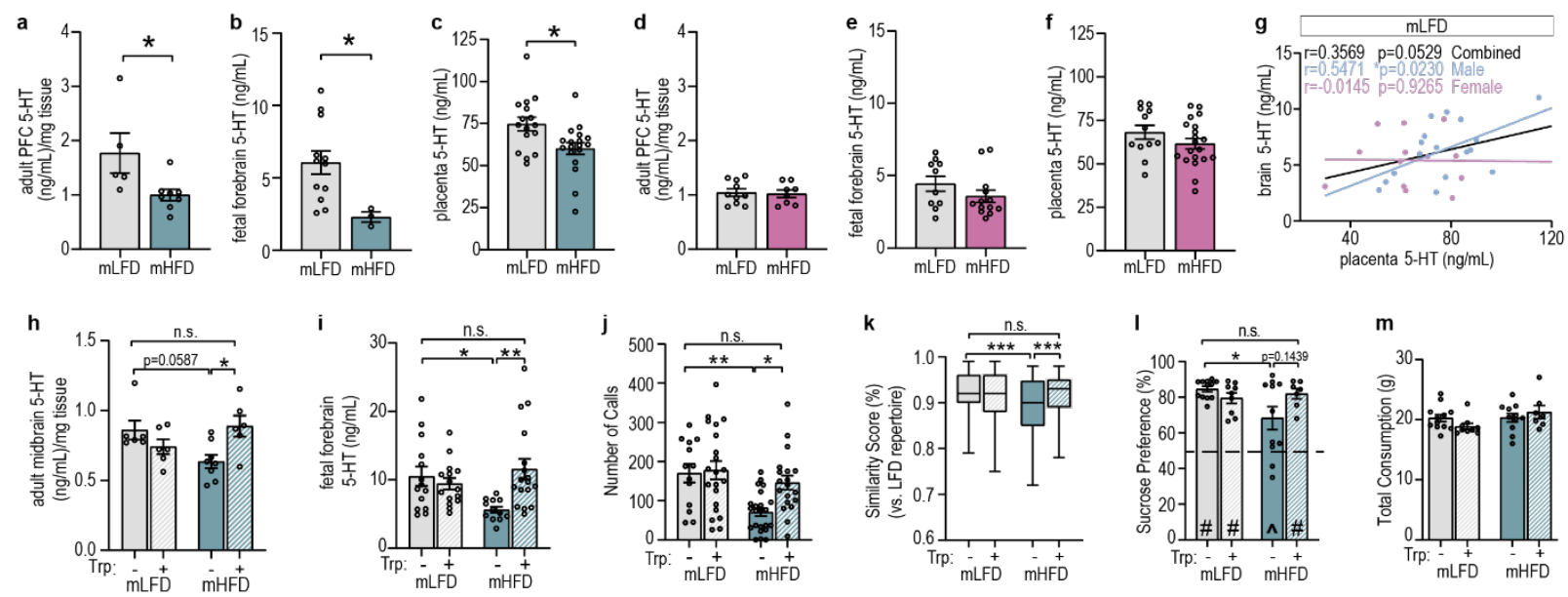

a, Adult male mHFD offspring have decreased prefrontal cortex (PFC) serotonin ( $n=5 \mathrm{mLFD}, 8 \mathrm{mHFD}$ male offspring) b, Male mHFD offspring have decreased fetal forebrain serotonin ( $n=12 \mathrm{mLFD}, 3 \mathrm{mHFD}$ male offspring). c, Male mHFD offspring have decreased placenta serotonin ( $n=16 \mathrm{mLFD}, 18 \mathrm{mHFD}$ offspring). d-f, mHFD does not impact adult ( $n=10 \mathrm{mLFD}, 8 \mathrm{mHFD}$ ) or fetal serotonin levels in female offspring (forebrain: $n=10 \mathrm{mLFD}, 13 \mathrm{mHFD}$; placenta: $n=12 \mathrm{mLFD}, 20 \mathrm{mHFD}$ female offspring) $\mathbf{g}$, Fetal serotonin levels are significantly correlated between brain and placenta in males only ( $\mathrm{n}=17 \mathrm{male}, 13 \mathrm{female} \mathrm{mLFD}$ offspring). $\mathrm{h}$, Maternal dietary tryptophan enrichment increases serotonin levels in mHFD adult male midbrain ( $\mathrm{n}=6 \mathrm{mLFD}, 6 \mathrm{mLFD}+\operatorname{Trp}, 8 \mathrm{mHFD}, 6 \mathrm{mHFD}+\mathrm{Trp}$ offspring) i, Maternal dietary tryptophan enrichment increases serotonin levels in mHFD fetal male forebrain ( $n=14 \mathrm{mLFD}, 15 \mathrm{mLFD}+\operatorname{Trp}, 12 \mathrm{mHFD}, 17 \mathrm{mHFD}+\operatorname{Trp}$ offspring) j-k, Maternal dietary tryptophan enrichment rescues mHFD-induced decrease in USV number and syllable repertoire similarity ( $\mathrm{n}=13 \mathrm{mLFD}, 21 \mathrm{mLFD}+$ Trp, $23 \mathrm{mHFD}, 19 \mathrm{mHFD}+\mathrm{Trp}$ offspring). I-m, Maternal dietary tryptophan enrichment increases sucrose preference in mHFD male offspring and does not impact total consumption ( $n=12 \mathrm{mLFD}, 9$ mLFD+Trp, $11 \mathrm{mHFD}, 7 \mathrm{mHFD}+$ Trp male offspring). Data are mean \pm s.e.m.; $n$ represents biologically independent mice. asterisk denoted $\mathrm{p}$-values are derived from unpaired two-tailed t-tests $(\mathbf{a}, \mathbf{b}, \mathbf{c})$, Pearson's correlation ( $\mathbf{g}$ ), or two-way ANOVA (fat content $\mathrm{x}$ tryptophan content; $\mathbf{h}, \mathbf{i}, \mathbf{j}, \mathbf{k}, \mathbf{I})$. other $\mathbf{p}$-values (\# p<0.0001, ^p<0.05) are derived from one-sample t-tests assessing difference from chance $(50 \%)$ (I). n.s. not significant.

studies demonstrating that placental serotonin correlates with fetal brain serotonin ${ }^{21}$, we

observed a positive correlation between placental and fetal forebrain serotonin in $\mathrm{mLFD}$

offspring (Fig 2g). Interestingly, the correlation was exclusively driven by male serotonin

levels. Importantly, mHFD disrupted the correlation between placental and brain serotonin

levels in males (Extended Data Fig 3a).

Quantitative real-time PCR ( $\mathrm{PPCR}$ ) showed a decrease in both the serotonin synthesis enzyme tryptophan hydroxylase 2 (Tph2) and the serotonin transporter (5HTT) in male mHFD placenta (Extended Data Fig 3b-c). We did not observe any changes in the expression of the alternate serotonin synthesis enzyme tryptophan hydroxylase 1 (Tph1), the rate-limiting enzyme indoleamine 2,3-dioxygenase (Ido1) that promotes the degradation of tryptophan through the kynurenine pathway, or monoamine oxidase $A$ $(M A O A)$, an enzyme responsible for the oxidative deamination of neurotransmitters, including serotonin, in male placentas (Extended Data Fig 3b). Interestingly, we did see 
a significant decrease in MAOA expression in female mHFD placenta, suggesting that an alternate neurotransmitter may be affected in female mHFD offspring, and that the placenta may play a critical role as a source for brain neurotransmitters other than serotonin (Extended Data Fig 3c).

To determine if the behavioral outcomes in male mHFD offspring were caused by diminished serotonin, we sought to rescue serotonin by supplementing the maternal lowand high-fat diets with tryptophan. Tryptophan is the precursor to serotonin, and placental serotonin levels are largely dependent on maternal tryptophan levels ${ }^{21,23}$. Tryptophan supplementation (+Trp; provided in LFD or HFD for the same duration as in Fig 1a) did not affect maternal weight gain, litter composition (i.e., size and sex ratio), or offspring weights (Extended Data Fig 3d-g). Maternal tryptophan supplementation did not change brain serotonin levels in adult mLFD offspring (Fig 2h; Extended Data Fig 3h). Interestingly, maternal tryptophan supplementation did not rescue serotonin levels in adult male mHFD PFC (Extended Data Figure 3h), whereas it did significantly increase serotonin levels in adult male mHFD midbrain (Fig $2 \mathrm{~h}$ ), where the majority of 5-HT neuronal cell bodies reside. Projections from the DRN to other brain regions, such as the ventral tegmental area (VTA), are also important for social behavior and reward, and future work investigating these circuits in the context of mHFD is needed ${ }^{25}$.

Maternal tryptophan supplementation increased placental serotonin levels in male mLFD offspring (Extended Data Fig 3i) in support of previous literature demonstrating that placental serotonin levels are dependent on maternal tryptophan levels. In male mHFD offspring, tryptophan supplementation was unable to substantially increase placental serotonin levels (Extended Data Fig 3i). Conversely, maternal tryptophan 
supplementation did increase fetal forebrain serotonin levels in male mHFD, but not mLFD offspring (Fig 2i). Further, Pearson correlation analyses demonstrated that maternal tryptophan enrichment disrupted the significant positive correlation between brain and placental serotonin levels in male mLFD offspring (Extended Data Fig 3j). No significant correlation was detected in mHFD (in agreement with our previous finding) or mHFD+Trp offspring (Extended Data Fig 3k). This suggests that in the context of mHFD and tryptophan enrichment, the fetal brain produces serotonin independently of the placenta. Together these data suggest that in males, placental serotonin levels are dependent on maternal tryptophan levels and can be disrupted by maternal diet, but fetal forebrain serotonin levels are not solely dependent on placental serotonin levels.

To determine if mHFD-induced behavioral deficits were rescued by maternal tryptophan supplementation, we assessed USVs and sucrose preference in male mLFD and mHFD offspring. Tryptophan supplementation did not impact USV or sucrose preference behavior in mLFD offspring (Fig 2j-m, Extended Data Fig3 I-m). However, maternal tryptophan supplementation fully rescued the decreased USV call number and similarity (Fig 2j-k), and partially rescued the deficits seen in total USV call time and mean call length (Extended Data Fig3 I-m), as well as the diminished sucrose preference in mHFD adults (Fig 2l-m). In all cases, mHFD+Trp offspring behavior was indistinguishable from that of mLFD or mLFD+Trp offspring. Maternal tryptophan enrichment did not alter female offspring brain serotonin levels or rescue female USV behavior (Extended Data Fig $3 n-s)$. This suggests that despite similar neonatal behavioral outcomes in male and female mHFD offspring, the mechanisms driving USV behavior in males and females may be distinct. In sum, these data demonstrate 1) a developmental role for serotonin in males 
that is perturbed by maternal high-fat diet, and 2) that maternal tryptophan enrichment is sufficient to restore high-fat diet-induced brain serotonin deficiencies and associated serotonin-dependent behaviors in males.

\section{mHFD induces TIr4-dependent inflammation driving offspring behavior changes}

Maternal HFD creates an environment of chronic inflammation that affects the developing fetus as well as the placenta ${ }^{26}$. Proinflammatory cytokines can promote tryptophan catabolism, effectively decreasing serotonin bioavailability ${ }^{27,28}$. Thus, we hypothesized that the maternal high-fat diet-induced inflammatory environment is translated to the fetus, and responsible for decreased serotonin in male mHFD offspring. We observed increased macrophage density in both male and female mHFD placenta (Fig 3a), as well as increased Iba1 and CD68 immunoreactivity (labeling microglia and phagosomes, respectively) in both male and female fetal brains (Extended Data Fig 4a, b). Microglia influence central circuits in numerous ways, such as by pruning excess synapses or phagocytosing excess or dying progenitor cells ${ }^{29-32}$. To investigate potential interactions between microglia and the developing central serotonin system, we reconstructed microglia from e14.5 dorsal raphe nuclei (DRN), where the majority of serotonergic cell bodies reside, and where 5-HT levels were decreased by mHFD and rescued by maternal tryptophan supplementation in males. IMARIS reconstructions revealed that male mHFD microglia in the DRN are significantly larger (volume) than mLFD microglia (Fig 3b). Further, serotonin signal comprised a higher percentage of phagosomes (CD68) in mHFD microglia versus mLFD (Fig 3b), demonstrating that microglia phagocytosis of serotonergic neurons may be increased in the context of mHFD, leading to decreased central serotonin bioavailability. Postnatal microglia express 
bioRxiv preprint doi: https://doi.org/10.1101/2021.11.12.468408; this version posted November 13, 2021. The copyright holder for this preprint (which was not certified by peer review) is the author/funder, who has granted bioRxiv a license to display the preprint in perpetuity. It is made available under aCC-BY-NC-ND 4.0 International license.

the serotonin receptor $5 \mathrm{HT} 2 \mathrm{~B}^{33}$ and alter their phagocytic capacity in response to serotonin ${ }^{34}$. Further, mice lacking microglial 5HT2B display prolonged neuroinflammation

in response to lipopolysaccharide (LPS), a classic agonist of macrophage toll-like receptor signaling ${ }^{35}$. Thus, we sought to investigate potential feedback between serotonin

levels and microglial phagocytosis of serotonin neurons. Microglial volume and serotonin phagocytosis were rescued in mHFD+Trp offspring, in parallel with 5-HT levels (Fig 3b;

Fig 3. mHFD induces TIr4-dependent inflammation driving offspring behavior changes
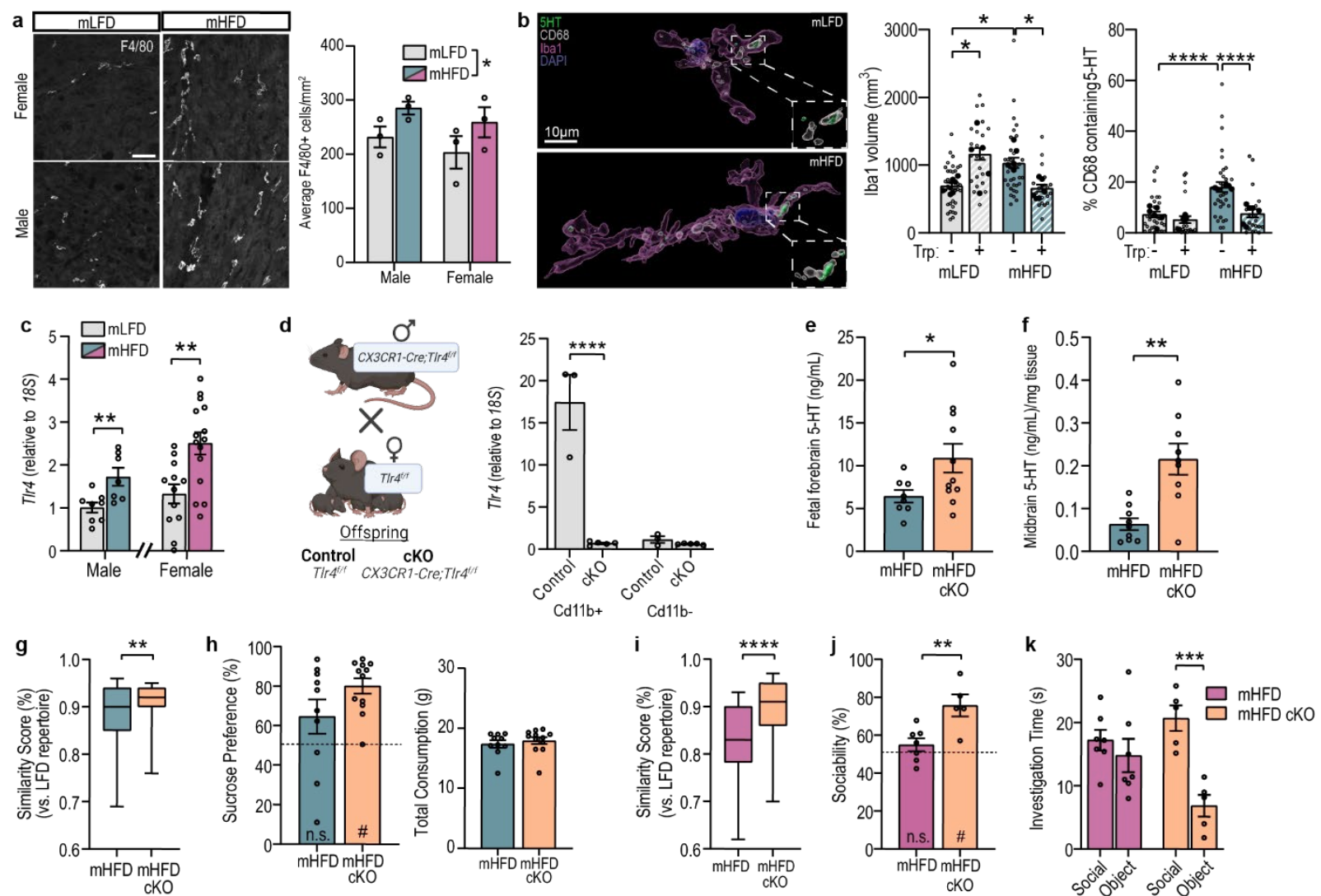

a, Increased macropahge density in male and female mHFD placenta ( $n=3$ animals per sex/diet). b, IMARIS reconstruction of DRN microglia reveals increased microglial volume and phagocytosis of serotonin in males at e14.5. Closed circles are animal average, open circles are individual microglia reconstructions ( $n=6$ mLFD, 5 mHFD mice); nested t-test. c, Increased TIr4 transcript expression in male and female mHFD placenta d, Schematic of macrophage-specific TIr4 knockout and confirmation of TIr4 knockdown in microglia ( $\mathrm{n}=3 \mathrm{control}, 5 \mathrm{TIr} 4 \mathrm{cKO}$ mice) e-f, Serotonin levels are significantly increased by macrophage-specific loss of TIr4 in male mHFD offspring fetal forebrain and adult midbrain ( $n=7$ mHFD control, 11 mHFD cKO fetal forebrain; $n=9$ mHFD, 9 mHFD cKO adult midbrain). g, Similary score is significantly increased by macrophage-specific TIr4 loss in male mHFD offspring ( $n=16$ mHFD, 15 mHFD cKO) $\mathbf{h}$, Male mHFD offspring lacking macrophage TIr4 signaling show a robust sucrose preference while mHFD male offspring with intact TIr4 signaling do not. Total consumption is unchanged $(n=10 \mathrm{mHFD}, 12 \mathrm{mHFD} \mathrm{cKO}) \mathbf{i}$, Similary score is significantly increased by macrophage-specific TIr4 loss in female mHFD offspring ( $n=6$ mHFD, 11 mHFD cKO) j-k, Social preference is resored in female mHFD offspring lacking macrophage-TIr4 signaling. mHFD female offspring lacking macrophage-TIr4 signaling investigate a social stimulus significantly more than an object, while female mHFD offspring with intact TIr4 signaling show no preference ( $n=7 \mathrm{mHFD}, 5 \mathrm{mHFD} \mathrm{cKO})$. Data are mean $\pm \mathrm{s}$.e. $\mathrm{m}$.; $n$ represents biologically independent mice. asterisk denoted $\mathrm{p}$-values are derived from unpaired two-tailed t-tests ( $\mathbf{c}, \mathbf{d}, \mathbf{e}, \mathbf{f}, \mathbf{g}, \mathbf{i}, \mathbf{j}, \mathbf{k})$ or two-way ANOVA (a,b). other p-values $(\# p<0.0001)$ are derived from one-sample t-tests assessing difference from chance $(50 \%)(h, j)$. 
see Figure $2 \mathrm{i}$ ), suggesting that in the context of mHFD, enrichment of 5 -HT levels is sufficient to prevent further aberrant microglia phagocytosis.

. The presence of serotonin receptors on placental macrophages, or the potential influence of serotonin on placental macrophages is unknown, although recent studies have demonstrated the presence of functional serotonin transporters in placental tissue ${ }^{36,37}$. Thus, we sought to investigate crosstalk between placental serotonin and placental macrophage density as well as fetal brain serotonin and microglia activity. Maternal tryptophan supplementation did not influence placental macrophage density in either mLFD or mHFD male offspring (Extended Data Fig 4c). Considering our finding that mLFD+Trp offspring had increased placental serotonin (see Extended Data Fig 3i), this suggests that placental macrophage density is not influenced by serotonin.

To investigate the potential pro-inflammatory pathways leading to increased placental macrophage and fetal brain microglia density, we assessed a panel of proinflammatory markers with qPCR (Extended Data Fig 4d). This revealed a specific increase in Toll-like receptor 4 (TIr4) in both males and females (Fig 3c). TIr4 is a pattern recognition receptor known to initiate pro-inflammatory signaling cascades in response to infection pathogens (e.g. bacterial, viral), and whose expression is increased in response to saturated fats/obesity ${ }^{38-41}$. Isolated fetal microglia and placental macrophages from obese dams have exaggerated cytokine production in response to lipopolysaccharide (LPS), a classic TIr4 agonist ${ }^{24}$. To determine if increased TIr4 signaling in macrophages was causal to decreased serotonin and serotonin-dependent behaviors in males, or to behavioral changes in females, we generated a conditional TIr4 knock-out mouse (cKO) line using Cx3cr1-Cre BAC-transgenic ${ }^{42}$ and TIr4 ${ }^{f / f}$ mice $^{43}$ (Fig 3d). Female control (TIr4f/f) 
mice were transitioned to HFD at four weeks-old for six weeks, then subsequently mated to male $\mathrm{Cx} 3 \mathrm{cr} 1-\mathrm{Cre} ; \mathrm{TI} 4^{f / f}$ mice to generate control and cKO mHFD offspring. qPCR revealed significant ( 95\%) knockdown of T/r4 in cKO microglia (Fig 3d). Excitingly, we saw significantly increased serotonin in male fetal forebrain and adult midbrain in mHFD cKO offspring (Fig 3e,f). Placental serotonin trended higher, but adult male mHFD cKO PFC serotonin was unchanged (Extended Data Fig 4e-f), consistent with our previous observation (see Fig 2). Increased fetal and adult midbrain serotonin was associated with increased USV similarity (Fig 3g) and sucrose preference (Fig 3h). Interestingly, TIr4 knockdown did not increase the number of USVs, total call time, or mean call length in male mHFD cKO offspring (Extended Data Fig 4g), suggesting that number of USVs and USV similarity may be driven by different mechanisms. Corroborating this, in female mHFD cKO offspring, we also saw an improvement of USV similarity (Fig 3i) but no change in the number of USVs, total call time, or mean call length (Extended Data Fig 4h). Juvenile female mHFD cKO offspring showed a significant improvement in social preference (Fig 3j,k), suggesting that mHFD-induced TIr4-dependent inflammation in females is responsible for behavioral outcomes, through a yet unknown mechanism.

Maternal overnutrition causes decreased prenatal serotonin in human male brain

\section{tissue}

To determine to what degree our findings are translatable to the human population, we obtained a large (37 individuals) cohort of matched tissues (fetal brain, fetal placenta, and maternal placenta (decidua)) from fetuses collected via elective termination at $72-82$ days post conception (d.p.c.), a timeframe that closely matches the embryonic developmental window used in our mouse studies (14.5-16.5 d.p.c.; Fig 4a). PCR for the 
Y chromosome (SRY; Extended Data Table 2) identified 17 male and 20 female fetal tissue sets, with the average age of 78.2 d.p.c. for both sexes (Extended Data Fig 5a). No maternal data were available, so we assessed decidual triglyceride accumulation to use as a proxy for maternal weight, given that women with overweight/obesity exhibit triglyceride accumulation in the placenta ${ }^{44}$. Triglyceride levels trended higher in pregnancies with female fetuses, but there was no significant difference between triglyceride accumulation in decidua associated with male or female pregnancies (Extended Data Fig 5b). Correlation of gene expression with maternal triglyceride accumulation from bulk RNA-seq from 16 matched male and 19 matched female brain and placenta samples revealed a strong dimorphic trend in gene expression. In male pregnancies, differentially correlated genes were predominantly over-expressed with increasing maternal weight (i.e. gene expression increased as maternal triglycerides increased); the opposite effect was seen in female pregnancies (Fig 4b). In the male brain, only 45 genes significantly correlated with maternal triglyceride accumulation (either positive or negative), whereas 781 genes were significantly correlated in females (Fig 4b). Gene Ontology (GO) Enrichment Analysis of genes significantly positively correlated with maternal triglyceride accumulation revealed enrichment of inflammatory signaling pathways in both male and female placenta (Fig 4c; full GO enrichment data is available in Extended Data Table 1). Correlation analyses of targeted genes reinforced these proinflammatory phenotypes; assessment of common macrophage, trophoblast and syncytiotrophoblast markers ${ }^{45}$ revealed specific gene associations predominantly within macrophages (Extended Data Fig 5c, Fig 4b). In males, GO enrichment from placental genes negatively correlated with maternal triglycerides revealed a pattern of repressive 
cell differentiation, particularly of immune cells (e.g. RUNX1 differentiation and myeloid cell differentiation). That is, with increasing maternal triglyceride accumulation, gene expression of factors important for cell differentiation decreased (Fig 4c). In females, placental genes negatively correlated with maternal triglycerides were often involved in vascular growth and development, suggesting that maternal triglyceride accumulation may decrease placental vasculogenesis/angiogenesis (Fig 4c), an important finding given that changes in the placental vascular network are associated with numerous pathologies, such as preeclampsia ${ }^{46,47}$. Inflammatory responses were additionally enriched in female brain (e.g. response to TGF- $\beta$ ), but too few genes were correlated in males to determine if this association was true independent of sex. We were unable to control for which brain region we sampled from, suggesting that regional differences may play a more dramatic role in brain inflammatory responses in males. Lastly, the top GO enrichment categories from female brain transcripts negatively correlated with maternal triglyceride accumulation involved neuronal development (Fig 4c), suggesting that maternal high-fat diet and accompanying fetal inflammation may impede brain development.

To determine if serotonin levels were impacted by maternal triglyceride accumulation, we assessed serotonin in human male and female fetal brain and placenta. Correlation analyses revealed a significant positive correlation between placental and brain serotonin only in male fetal tissues (Fig 4d). This is consistent with previous literature (sex not reported) ${ }^{21}$ and our findings in male mice (see Fig $2 \mathrm{~g}$ ). Correlation analyses further revealed a negative correlation between brain or placental serotonin levels and maternal triglyceride accumulation in male tissue only (Fig 4e). This is in agreement with our findings in mice that a maternal high-fat diet results in decreased 
bioRxiv preprint doi: https://doi.org/10.1101/2021.11.12.468408; this version posted November 13, 2021. The copyright holder for this preprint (which was not certified by peer review) is the author/funder, who has granted bioRxiv a license to display the preprint in perpetuity. It is made available under aCC-BY-NC-ND 4.0 International license.

brain and placental serotonin levels in male fetal tissue (see Fig 2b,c). In sum, in both mice and humans, maternal high-fat diet results in the perpetuation of inflammation to the fetal brain and placenta in both sexes. In males, this inflammatory response results in aberrant phagocytosis of serotonin neurons and long-lasting decreased levels of brain serotonin and decreased reward behavior.

Fig 4. Maternal overnutrition causes decreased prenatal serotonin in human male brain tissue

a

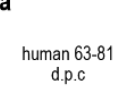

b
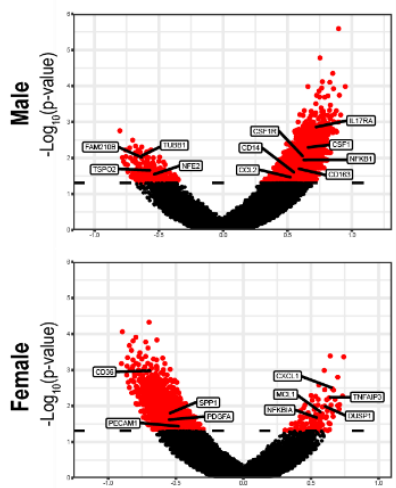

Pearson's Correlation Coefficient (TPM correlated with maternal triglycerides)
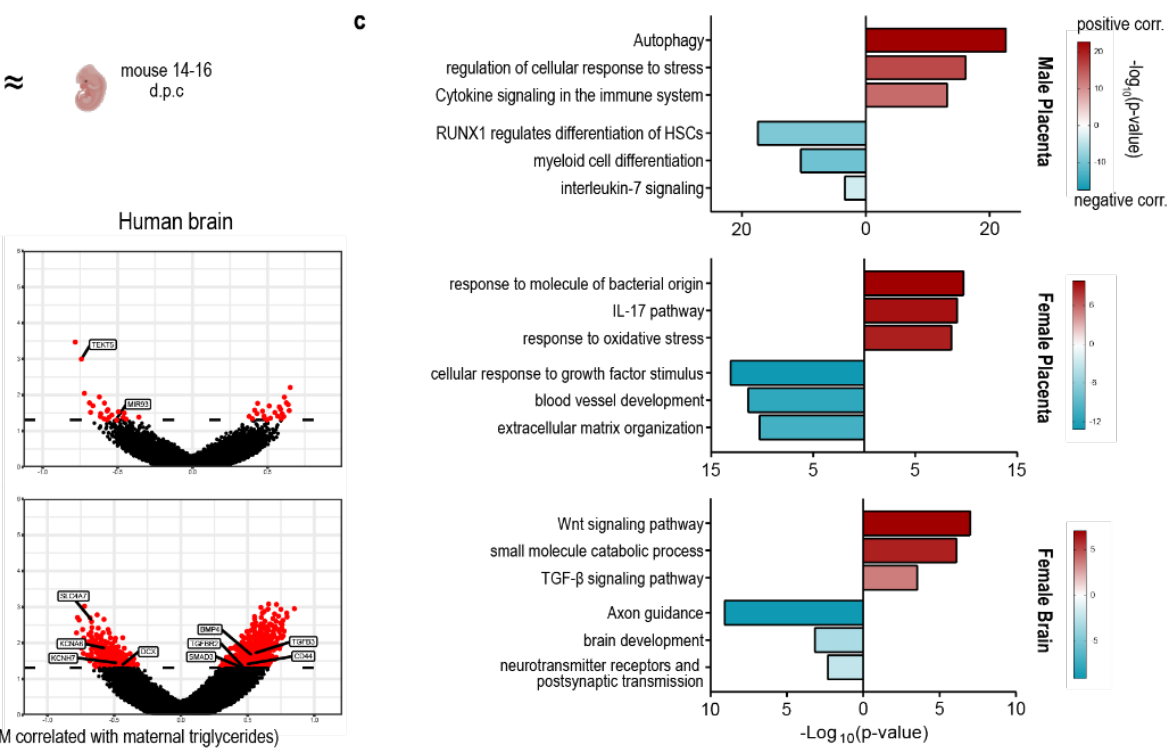

Male

d
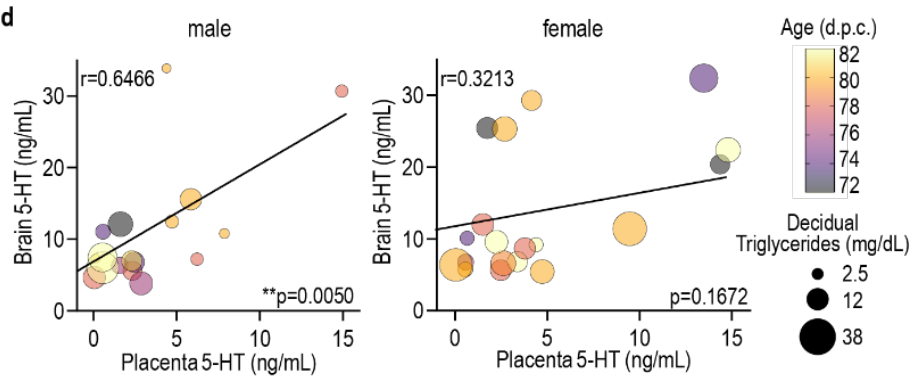
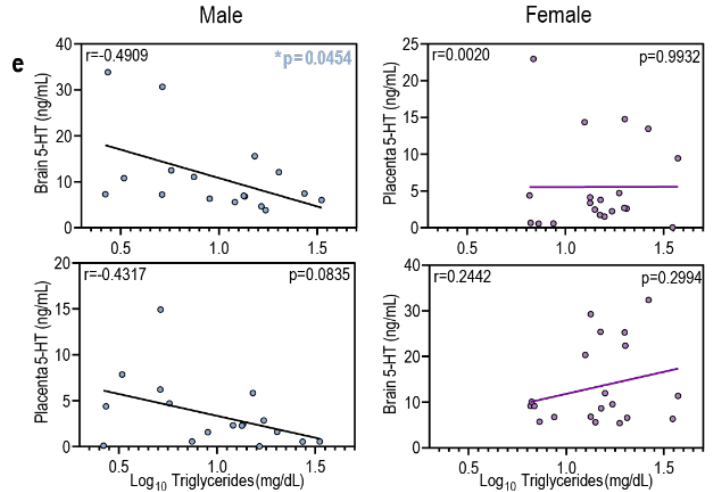

a, Human fetal development at 63-81 days post conception (d.p.c.) is roughly equivalent to mouse embryonic development at 14-16 d.p.c. b, Scatter plots showing transcript (TPM) correlation with maternal triglycerides versus - $\log _{10}(\mathrm{p}$-value) in male and female placenta and brain bulk sequencing data ( $\mathrm{n}=16$ matched male tissue sets, 19 female). Red dots indicate a significant $p$-value $(<0.05)$. c, GO Enrichment analysis of transcripts significantly positively correlated with maternal triglyceride accumulation demonstrates a robust upregulation in immune responses in male placenta. GO Enrichment analysis of transcripts significantly positively correlated with maternal triglyceride accumulation in placenta shows increased immune responses. GO enrichment of negatively correlated with maternal triglyceride accumulation in maternal triglyceride accumulation in placenta shows increased immune responses. GO enrichment of negatively correlated with maternal triglyceride accumulation in
the brain shows downregulation of neuronal development and function, as well as oxytocin signaling. d, Multivariate dot plots show Pearson correlations between brain and placental serotonin from male and female human fetal tissues. Dot color represents the age of the matched tissue set, and dot size represents the maternal decidual triglyceride level (mg/dL), a proxy for maternal dietary fat intake. Brain and placental serotonin levels are significantly positively correlated in male tissues between 72 and 82 d.p.c. Brain and placental serotonin levels are not correlated in female tissues ( $n=17$ matched male tissue sets, 20 female). e, Brain serotonin levels are significantly negatively correlated with decidual triglyceride levels in males only. Placental serotonin levels trend towards a negative correlation with decidual triglycerides in males only. Data are mean \pm s.e.m.; $n$ represents biologically independent matched human tissue set. asterisk denoted p-values are derived from Pearson's correlations. 


\section{Discussion}

Here, we demonstrate a fundamental, sex-biased mechanism through which maternal high-fat diet increases offspring susceptibility to neurological disorder development (Extended Data Fig 5c). Our results demonstrate that, in the context of maternal high-fat diet, in utero inflammation is mediated by the pattern recognition receptor TIr4 in males and females, leading to increased macrophage reactivity in both the placenta and the fetal brain. In female mice, TIr4-dependent inflammation causes diminished social preference through a serotonin-independent mechanism. In humans, maternal decidual triglyceride accumulation is negatively associated with brain development, suggesting that Tlr4-dependent inflammation impacts neuronal development in females, though the target neuronal population is not yet known. In male mHFD offspring, embryonic microglia aberrantly phagocytose serotonin neurons in the DRN, leading to diminished brain serotonin from embryonic stages through adulthood, and offspring anhedonia. In humans, maternal decidual triglyceride accumulation is associated with pro-inflammatory signaling pathways in both sexes, and negatively correlated with brain serotonin levels in males only, reinforcing our findings in mice.

Serotonin dysregulation is heavily implicated in neurological disorders such as depression. There is an urgent need to investigate sex differences in depression given that it is a female-biased disorder with sex differences in presentation and treatment efficacy $^{48}$. Further, likelihood of postpartum depression in women is increased with male versus female pregnancies ${ }^{49}$. Despite decades of research, treatments for anxiety and depression in both sexes have limited efficacy. Selective serotonin reuptake inhibitors (SSRIs), serotonin-norepinephrine reuptake inhibitors (SNRIs), and tricyclic 
antidepressants (TCAs) - which also block serotonin and norepinephrine reuptake - are the primary pharmacological treatments for depression, yet symptom relief can take weeks, if not months, and only about $30 \%$ of individuals report symptom relief at all ${ }^{48}$. Recent literature has demonstrated multiple potential anti-inflammatory actions of SSRIs and SNRIs in addition to their actions on monoamine reuptake ${ }^{48}$. In particular, the SSRI fluoxetine has been demonstrated to prevent increased TLR4 protein levels in a rodent model of chronic social defeat stress-induced depression ${ }^{50}$. Together, the literature demonstrates that there are robust sex differences in depression, and that depression pathology is likely mediated by a combination of factors, including a combination of diminished serotonin bioavailability and increased neuroinflammation. Here, we find a male-specific decrease in serotonin driven by maternal high-fat diet-induced inflammation during embryonic development. To date, the majority of research implicating serotonin in depression has been done in adults; our results suggest that the development of the serotonin system, and in particular reciprocal interactions between serotonergic neurons and the neuroimmune system, are critical for adult serotonin levels and related behavioral outcomes. Our results help shed light on developmental sex differences increasing susceptibility to depression and could help explain the lackluster efficacy of SSRI treatment, particularly in women.

Seminal work reported the placenta as a transient source of serotonin for the fetal brain $^{21}$, though recent studies suggest that the fetal placenta lacks the enzymes required for serotonin synthesis ${ }^{37}$. No studies have investigated whether fetal sex plays a role in serotonin synthesis or transfer in the placenta. Further, whether developmental serotonin levels are reflective of adult serotonin levels, and if disruptions to serotonin levels during 
this window impact the brain long-term are unknown. Here, we show that fetal placenta and brain serotonin levels are susceptible to perinatal inflammation in males only, and that decreased fetal serotonin levels are reflective of decreased adult brain serotonin levels in the context of maternal high-fat diet. We further found that transcript levels of both the neuronal serotonin synthesis enzyme and serotonin transporter (Tph2 and 5HTT, respectively) were decreased in male mHFD placenta, but we did not distinguish between the maternal and fetal compartment of the placenta. By manipulating fetal serotonin levels through maternal dietary tryptophan enrichment, we can shed light on the relationship between placental and fetal brain serotonin. While at baseline there is a significant positive correlation between placental and fetal brain serotonin in males only, maternal tryptophan supplementation disrupted the correlation. This suggests that either the placenta is not the sole source of fetal brain serotonin, or that the fetal brain is capable of sensing and limiting the influx of placental serotonin in cases of excess. Careful investigation of the spatiotemporal expression of placental serotonin transport and synthesis enzymes in male and female placenta and decidua is warranted to further clarify this topic.

Serotonin has been postulated to influence macrophage phagocytic activity and cytokine production for decades ${ }^{51,52}$. Here, we demonstrate a novel male-specific potential feedback loop through which microglial phagocytosis influences central serotonin levels, which in turn influence microglia phagocytic activity. mHFD results in increased TIr4-dependent inflammation in macrophages, which results in microglia overphagocytosis of serotonin neurons in the dorsal raphe nucleus in males only. Considering serotonin is thought to decrease phagocytic capacity ${ }^{34}$, this resulting decrease in 
serotonin thus propagates the over-phagocytosis of the mHFD microglia. Both abrogation of overactive TIr4-signaling in macrophages and increased serotonin bioavailability via maternal tryptophan supplementation were sufficient to rescue central serotonin levels, demonstrating the reciprocal relationship between inflammation and serotonin in the male fetal brain. Interestingly, we saw that despite tryptophan enrichment having no effect on fetal brain serotonin in mLFD offspring, mLFD+Trp microglia were larger than mLFD microglia. Microglia are known to catabolize tryptophan to the neurotoxin quinolinic acid ${ }^{53}$, and excess quinolinic acid can propagate inflammation ${ }^{54}$. Together with our data, this suggests that maternal dietary tryptophan in excess could have negative consequences for microglial morphology or function.

In closing, maternal overweight/obesity is becoming exceedingly prevalent, particularly in developed countries. In the United States, it is estimated that greater than $50 \%$ of women are diagnosed as overweight or obese at their first prenatal visit ${ }^{5}$. High maternal weight creates an environment of chronic inflammation and is associated with numerous negative outcomes, both maternal and fetal ${ }^{1-4}$. Despite this, mechanisms through which chronic maternal inflammation driven by high maternal weight impact offspring neurodevelopment are scarce. In our mouse model, we demonstrate that fetal inflammation is propagated by macrophage-specific TIr4 in both male and female mHFD offspring, along with sex-specific neurodevelopmental and behavioral outcomes. Further, we generated a large sequencing dataset of human fetal tissue from both male and female pregnancies, which demonstrated 1) a strong degree of fetal inflammation in both male and female placenta, and particularly in female brain in response to increasing maternal triglyceride accumulation, and 2) sex-specific inflammatory responses. These 
data will shed light on what other fetal inflammatory pathways might be responsive to maternal triglyceride accumulation and will provide direction for future studies.

\section{Methods}

Animals: All procedures relating to animal care and treatment conformed to Duke Institutional Animal Care and Use Committee (IACUC) and NIH guidelines. Animals were group housed in a standard 12:12 light-dark cycle. The following mouse lines were used in this study: C57BL/6J (Jackson Laboratory, stock no. 000664), Cx3cr1-Cre BACtransgenic ${ }^{42}$, T/r4/f $(\mathrm{JAX}$ 02487243). Cx3cr1-Cre was maintained in males for all experimental studies.

Special Diets: Female C57BL/6J mice were placed on special diet at 4-weeks old and given ad libitum access to their assigned diet chow and water. Weight gain was assessed three times/week. After 6-weeks on diet, females were mated with $C 57 B L / 6 \mathrm{~J}$ males. Pregnancy was determined by the presence of a copulation plug (gestational day 0.5) and maternal weight was assessed daily. Dams were maintained on their assigned diet chow throughout gestation and until the pups were weaned. At weaning, offspring were group housed with sex-matched littermates and provided ad libitum access to rodent chow 5001 (standard rodent chow, 13\% kcal from fat). Low-fat, high-fat, lowfat+tryptophan, and high-fat+tryptophan diets were purchased from Research Diets (low fat: D12450Hi, high-fat: D12451i). Tryptophan enriched diets were custom formulations resulting in $1 \%$ final tryptophan content.

Tissue Collection and Analyses: Murine Tissue collection: Pregnant dams were euthanized using $\mathrm{CO}_{2}$ following Duke University Animal Care and Use guidelines. Uterine 
horns were dissected and placed on ice in sterile 1X PBS. Individual embryos were separated, and placenta, brain, and tail tissue (for genotyping) were collected. Placenta were dissected into three equal pieces. One piece was flash-frozen in liquid nitrogen at stored at $-80^{\circ} \mathrm{C}$ until ELISA. One piece was placed into $4 \%$ paraformaldehyde in PBS (PFA, Sigma) for immunohistochemistry (IHC). The final piece was placed in TriZol and stored at $-80^{\circ} \mathrm{C}$. Dissected fetal brains were cut in half coronally; fetal forebrain tissue was flash frozen for ELISA while the remainder of the brain was placed into $4 \%$ PFA for IHC. For adult brain collections, offspring were anesthetized with $\mathrm{CO}_{2}$ and transcardially perfused with saline. Further dissections were done to isolate prefrontal cortex (PFC) and midbrain, and brain tissues were immediately flash frozen in liquid nitrogen and stored at $-80^{\circ} \mathrm{C}$ until processing.

Human tissue collection: Human placenta (maternal decidua separated from fetal placenta) and fetal brain tissues were obtained from the NIH-supported Laboratory of Developmental Biology at the University of Washington between the years 1999 and 2010 and used under a protocol approved by the Duke University Institutional Review Board (Pro00014066). Tissue specimens were stored at $-80^{\circ} \mathrm{C}$ until required for RNA or protein extraction. Prior to collection, informed consent was obtained from all individuals undergoing the elective termination.

Immunohistochemistry: Placenta and brain tissue were fixed in $4 \%$ PFA overnight at $4{ }^{\circ} \mathrm{C}$, cryoprotected in $30 \%$ sucrose $+0.1 \%$ sodium azide in PBS (Sigma), and embedded in OCT (Sakura Finetek, Torrance, CA) before being cryo-sectioned directly onto Superfrost slides (Fisher) at $40 \mu \mathrm{m}$. Sections were frozen at $-20^{\circ} \mathrm{C}$ for storage. For IHC, sections were permeabilized in 1\% Triton X-100 in PBS for 12 minutes, blocked at room temperature 
(RT) with $5 \%$ goat serum (GS) in PBS $+0.1 \%$ Tween-20, then incubated for 2 nights at $4^{\circ} \mathrm{C}$ with chicken anti-Iba1 (Synaptic Systems, 234 006) rat anti-CD68 (Biolegend, 137002), and rabbit anti-serotonin (Sigma, S5545) or rat anti-F4/80 (Abcam, ab6640). Sections were then washed with PBS and incubated in the following secondary antibodies: anti-rabbit Alexa-488, anti-chicken Alexa-647, and anti-rat Alexa-568 (brain), or anti-rat Alexa-568 (placenta; 1:200; ThermoFisher), and DAPI $(100 \mu \mathrm{g} / \mathrm{mL})$. Ten zstacks of $0.5 \mu \mathrm{m}$ optical thickness were taken at 20X using a Zeiss Axiolmager.M2 (with ApoTome.2) from at least 5 sections (each $400 \mu \mathrm{m}$ apart) from the fetal compartment of each placenta for $\mathrm{F} 4 / 80+$ cell quantification. Ten z-stacks of $0.5 \mu \mathrm{m}$ optical thickness were also taken at 20X from at least 3 fetal brain sections/animal (each section being $200 \mu \mathrm{m}$ apart) for Iba1 and CD68 immunoreactivity. For IMARIS reconstructions, z-stacks of $0.5 \mu \mathrm{m}$ optical thickness were taken to capture entire microglia. Surface reconstructions of Iba1, CD68, 5HT, and DAPI were done for at least 19 microglia/diet from $6 \mathrm{mLFD}$, 5mLFD+Trp, 5 mHFD, and 6 mHFD+Trp male offspring.

Serotonin ELISA: Mouse placenta and brain tissue were thawed on ice and homogenized in freshly prepared lysis buffer (final concentrations: $1 \mathrm{mg} / \mathrm{mL}$ ascorbic acid, $0.147 \% \mathrm{NP}$ 40, 1X HALT Protease Inhibitor (Thermo 78443) in 1X PBS). Samples were then centrifuged at top speed for 20 minutes; supernatant was diluted 1:2 for placenta, or undiluted for fetal brain preparations, and loaded and processed according to manufacturer instructions (Enzo, ADI-900-175).

Decidual Triglycerides: Decidua were thawed on ice, and $\sim 150 \mathrm{mg}$ tissue was homogenized in $1 \mathrm{~mL}$ NP40 Substitute Assay Reagent and processed (undiluted) 
according to manufacturer instructions at room temperature (Cayman Chemical, 10010303).

Behavior: Ultrasonic Vocalizations (USVs): At P8, pups were brought in their home cage (with the dam being the only adult in the cage) to the testing room. Each pup was removed from their home cage and placed directly in a small cup in an insulated chamber with an AviSoft Condenser ultrasound microphone (Avisoft-Bioacoustics CM16/CMPA) suspended four inches above the contained pup. After 3 minutes of recording, pups were weighed and sexed before being returned to their home cage. After the dam attended to each returned pup, the home cage was returned to the colony room. USV analysis was done with Avisoft and MUPET ${ }^{13}$ (Mouse Ultrasonic Vocalization Extraction Tool). Default MUPET parameters were used with the exception of: noise-reduction $=8.8$, minimumsyllable-duration $=2.0$, maximum-syllable-duration $=200.0$, minimum-syllable-totalenergy $=-15.0$, minimum-syllable-peak-amplitude $=-25.0$, and minimum-syllabledistance $=5.0$.

Open Field Test: Adult offspring were placed in a $50 \mathrm{~cm} \times 50 \mathrm{~cm}$ square enclosure with $38 \mathrm{~cm}$ high walls and allowed to explore freely for ten minutes ${ }^{55}$. Center-avoidance was assessed by comparing time spent at the periphery to time spent centrally/number of central entries (middle third of chamber). Behaviors were recorded and analyzed using EthoVision video tracking software (Noldus).

Sociability: One day prior to testing, subject and stimulus animals were habituated to the testing room for a minimum of 1 hour before being individually habituated to the 3 chambered social preference boxes for 5 minutes each. On test day, subject and stimulus animals were habituated to the testing room for a minimum of one hour before testing. 
Juvenile offspring were tested for preference to interact with either a novel age- and sexmatched conspecific or an inanimate novel object (rubber duck). Mice were placed in the middle chamber with the inanimate object confined on one side of the test and a novel conspecific confined on the other side for 5 minutes. All behavior was recorded and quantified using Solomon Coder by an observer blind to sex/treatment. Social preference is represented by a sociability score significantly higher than chance $(50 \%)$. Sociability score formula: (time investigating social stimulus/ (total investigation time (social + object) $\left.{ }^{*} 100\right)$.

Sucrose Preference: Adult offspring were tested for their preference for a $1 \%$ sucrose solution in water or plain water. Mice were provided with 2 drinking bottles for a total of 6 days. After 3 days (acclimation to 2 bottles with drinking water in both), one bottle was filled with a $1 \%$ sucrose solution while the other was filled with drinking water. Sucrose versus water intake was measured daily over the next 3 days. The positions of the bottles were switched daily to reduce any bias in side-preference. Sucrose preference is calculated as a percentage of the volume of $1 \%$ sucrose intake over total fluid intake averaged over 3 days.

qRT-PCR: RNA was isolated using TRIzol RNA extraction reagent chloroform extraction. Either $1 \mu \mathrm{g}$ (placenta) or 200ng (isolated microglia) RNA was reverse-transcribed into cDNA using a Qiagen QuantiTect Reverse Transcription kit (205311). qPCR was performed on an Eppendorf Realplex ep Mastercycler using Sybr/Rox amplification with a QuantiFAST PCR kit (204056). Primer sequences are listed in Extended Data Table 2. Fold change was calculated using the $2^{(-\Delta \Delta C T)}$ method with $18 \mathrm{~S}$ as an endogenous control. Sequencing: RNA from human tissue was prepared for sequencing using an Illumina TrueSeq RNA Exome kit and sequenced on an Illumina NovaSeq 6000 configured for a S2 
flow cell at 50bp PE. Brain and placenta count matrices were processed separately. Reads were aligned to the human genome using Bowtie $2^{56}$. Transcripts per million (TPM) was calculated on the raw counts and used for all subsequent analyses. Gene inclusion was determined using EgeR ${ }^{57}$ (threshold of 1). Pearson's correlation coefficients and p-values were calculated for each gene (TPM correlated with log10(maternal triglyceride accumulation)), and data was plotted in a volcano plot using custom $\mathrm{R}$ script. All code for processing is currently hosted and publicly available (https://github.com/bendevlin18/humanfetal-RNASeq). Gene Ontology (GO) enrichment analysis (Metascape ${ }^{58}$ ) was performed on genes with significant positive and negative correlations separately $(p<0.05)$. All data will be publicly available; GEO Accession number pending.

Acknowledgements: We thank the Duke University School of Medicine for the use of the Sequencing and Genomic Technologies Shared Resource, which provided library preparation and sequencing service. We also thank Kristina Sakers for guidance and $\mathrm{R}$ scripts for sequencing analysis.

Funding: Research reported in this publication was supported by the Eunice Kennedy Shriver National Institute of Child Health \& Human Development (F32HD104430 to A.M.C.), the National Institute of Environmental Health Sciences (R01 ES025549 to S.D.B.), the Robert and Donna Landreth Family Foundation, and the Charles Lafitte Foundation.

Author Contributions: S.D.B, L.S., and J.B. conceived the study and, together with A.M.C, designed the experiments. A.M.C, B.A.D, YC.J, C.H., B.P., K.R.W., F.J., A.B.C- 
S, and E.R.L. performed experiments and data analysis. A.M.C. wrote the manuscript with contributions from all of the authors.

The authors declare no competing interests.

${ }^{*}$ Correspondence and requests for materials should be addressed to Staci D. Bilbo (staci.bilbo@duke.edu)

Supplementary Information is available for this paper.

Extended Data Table 1. Raw GO enrichment analysis results

Extended Data Table 2. PCR Primer sequences

1. Vickers, M. H., Breier, B. H., Cutfield, W. S., Hofman, P. L. \& Gluckman, P. D. Fetal origins of hyperphagia, obesity, and hypertension and postnatal amplification by hypercaloric nutrition. Am J Physiol Endocrinol Metab 279, E83-7 (2000).

2. Edlow, A. G. Maternal obesity and neurodevelopmental and psychiatric disorders in offspring. Prenat Diagn 37, 95-110 (2017).

3. Kott, J. \& Brummelte, S. Trick or treat? Evaluating contributing factors and sex-differences for developmental effects of maternal depression and its treatment. Horm Behav 111, 31-45 (2019).

4. Krakowiak, P. et al. Maternal metabolic conditions and risk for autism and other neurodevelopmental disorders. Pediatrics 129, e1121-8 (2012).

5. Deputy, N., Dub, B. \& Sharma, A. J. Prevalence and Trends in Prepregnancy Normal Weight -48 States, New York City, and District of Columbia, 2011-2015. MMWR Morb Mortal Wkly Rep 66, 14021407 (2019). 
6. Altemus, M., Sarvaiya, N. \& Epperson, C. N. Sex differences in anxiety and depression clinical perspectives. Front Neuroendocrinol 35, 320-30 (2014).

7. McLean, C. P., Asnaani, A., Litz, B. T. \& Hofmann, S. G. Gender Differences in Anxiety Disorders: Prevalence, Course of IIIness, Comorbidity and Burden of Illness. J Psychiatr Res 45, 1027-35 (2011).

8. Kessler, R. C. et al. Lifetime and 12-month prevalence of DSM-III-R psychiatric disorders in the United States. Results from the National Comorbidity Survey. Arch Gen Psychiatry 51, 8-19 (1994).

9. Cox, K. H. \& Rissman, E. F. Sex differences in juvenile mouse social behavior are influenced by sex chromosomes and social context. Genes Brain Behav 10, 465-472 (2011).

10. An, X.-L. et al. Strain and sex differences in anxiety-like and social behaviors in C57BL/6J and BALB/cJ mice. Exp Anim 60, 111-123 (2011).

11. Scattoni, M. L., Crawley, J. \& Ricceri, L. Ultrasonic vocalizations: a tool for behavioural phenotyping of mouse models of neurodevelopmental disorders. Neurosci Biobehav Rev 33, 508-515 (2009).

12. Yin, X. et al. Maternal Deprivation Influences Pup Ultrasonic Vocalizations of C57BL/6J Mice.

PLoS One 11, (2016).

13. Van Segbroeck, M., Knoll, A. T., Levitt, P. \& Narayanan, S. MUPET-Mouse Ultrasonic Profile ExTraction: A Signal Processing Tool for Rapid and Unsupervised Analysis of Ultrasonic Vocalizations. Neuron 94, 465-485.e5 (2017).

14. Mun, H.-S., Lipina, T. V. \& Roder, J. C. Ultrasonic Vocalizations in Mice During Exploratory Behavior are Context-Dependent. Frontiers in Behavioral Neuroscience 9, 316 (2015).

15. Mosienko, V., Beis, D., Alenina, N. \& Wöhr, M. Reduced isolation-induced pup ultrasonic communication in mouse pups lacking brain serotonin. in Mol Autism vol. 6 (2015).

16. Sachs, B. D., Ni, J. R. \& Caron, M. Sex differences in response to chronic mild stress and congenital serotonin deficiency. Psychoneuroendocrinology 40, 123-9 (2014). 
17. Garcia-Garcia, A. L. et al. Serotonin signaling through prefrontal cortex 5-HT1A receptors during adolescence can determine baseline mood-related behaviors. Cell Rep 18, 1144-56 (2017).

18. C, C., SG, B. \& O, B. Optogenetic modulation of descending prefrontocortical inputs to the dorsal raphe bidirectionally bias socioaffective choices after social defeat. Frontiers in behavioral neuroscience 8, (2014).

19. Nishizawa, S. et al. Differences between males and females in rates of serotonin synthesis in human brain. in Proc Natl Acad Sci U S A vol. 94 5308-13 (1997).

20. Renoir, T. et al. Sexually Dimorphic Serotonergic Dysfunction in a Mouse Model of Huntington's Disease and Depression. in PLoS One vol. 6 (2011).

21. Bonnin, A. et al. A transient placental source of serotonin for the fetal forebrain. Nature $\mathbf{4 7 2}$, $347-350$ (2011).

22. Song, L. et al. Prenatal high-fat diet alters placental morphology, nutrient transporter expression, and mtorc1 signaling in rat. Obesity (Silver Spring) 25, 909-919 (2017).

23. Goeden, N. et al. Maternal Inflammation Disrupts Fetal Neurodevelopment via Increased Placental Output of Serotonin to the Fetal Brain. J. Neurosci. 36, 6041-6049 (2016).

24. Edlow, A. G. et al. Placental Macrophages: A Window Into Fetal Microglial Function in Maternal Obesity. International Journal of Developmental Neuroscience S0736574818302508 (2018) doi:10.1016/j.ijdevneu.2018.11.004.

25. Zou, W.-J. et al. A discrete serotonergic circuit regulates vulnerability to social stress. Nat Commun 11, 4218 (2020).

26. Roberts, K. A. et al. Placental structure and inflammation in pregnancies associated with obesity. Placenta 32, 247-54 (2011). 
27. Babcock, T. A. \& Carlin, J. M. Transcriptional activation of indoleamine dioxygenase by

interleukin 1 and tumor necrosis factor alpha in interferon-treated epithelial cells. Cytokine 12, 588-

94 (2000).

28. O'Connor, J. C. et al. Interferon-gamma and tumor necrosis factor-alpha mediate the

upregulation of indoleamine 2,3-dioxygenase and the induction of depressive-like behavior in mice in response to bacillus Calmette-Guerin. J Neurosci 29, 4200-9 (2009).

29. Cunningham, C. L., Martinez-Cerdeno, V. \& Noctor, S. C. Microglia regulate the number of neural precursor cells in the developing cerebral cortex. J Neurosci 33, 4216-33 (2013).

30. Block, C. L. et al. Prenatal Environmental Stressors Impair Postnatal Microglia Function and Adult Behavior in Males. 2020.10.15.336669

https://www.biorxiv.org/content/10.1101/2020.10.15.336669v2 (2020) doi:10.1101/2020.10.15.336669.

31. Kopec, A. M., Smith, C. J., Ayre, N. R., Sweat, S. C. \& Bilbo, S. D. Microglial dopamine receptor elimination defines sex-specific nucleus accumbens development and social behavior in adolescent rats. Nature Communications 9, 1-16 (2018).

32. VanRyzin, J. W. et al. Microglial Phagocytosis of Newborn Cells Is Induced by Endocannabinoids and Sculpts Sex Differences in Juvenile Rat Social Play. Neuron 102, 435-449.e6 (2019).

33. Kolodziejczak, M. et al. Serotonin Modulates Developmental Microglia via 5-HT2B Receptors: Potential Implication during Synaptic Refinement of Retinogeniculate Projections. ACS Chem Neurosci 6, 1219-1230 (2015).

34. Krabbe, G. et al. Activation of serotonin receptors promotes microglial injury-induced motility but attenuates phagocytic activity. Brain Behav Immun 26, 419-28 (2012).

35. Béchade, C. et al. The serotonin 2B receptor is required in neonatal microglia to limit neuroinflammation and sickness behavior in adulthood. Glia 69, 638-654 (2021). 
36. Baković, P. et al. Differential Serotonin Uptake Mechanisms at the Human Maternal-Fetal Interface. Int J Mol Sci 22, 7807 (2021).

37. Kliman, H. J. et al. Pathway of Maternal Serotonin to the Human Embryo and Fetus.

Endocrinology 159, 1609-1629 (2018).

38. Medzhitov, R. Toll-like receptors and innate immunity. Nat Rev Immunol 1, 135-145 (2001).

39. Reyna, S. M. et al. Elevated Toll-Like Receptor 4 Expression and Signaling in Muscle From Insulin-

Resistant Subjects. Diabetes 57, 2595-2602 (2008).

40. Shi, H. et al. TLR4 links innate immunity and fatty acid-induced insulin resistance. J Clin Invest

116, 3015-3025 (2006).

41. Milanski, M. et al. Saturated Fatty Acids Produce an Inflammatory Response Predominantly through the Activation of TLR4 Signaling in Hypothalamus: Implications for the Pathogenesis of Obesity. Journal of Neuroscience 29, 359-370 (2009).

42. Rivera, P. D. et al. Removal of microglial-specific MyD88 signaling alters dentate gyrus doublecortin and enhances opioid addiction-like behaviors. Brain, Behavior, and Immunity 76, 104115 (2019).

43. McAlees, J. W. et al. Distinct TIr4-expressing cell compartments control neutrophilic and eosinophilic airway inflammation. Mucosal Immunol 8, 863-873 (2015).

44. Desoye, G. \& Wells, J. C. K. Pregnancies in Diabetes and Obesity: The Capacity-Load Model of Placental Adaptation. Diabetes 70, 823-830 (2021).

45. Liu, Y. et al. Single-cell RNA-seq reveals the diversity of trophoblast subtypes and patterns of differentiation in the human placenta. Cell Res 28, 819-832 (2018).

46. Wadsack, C., Desoye, G. \& Hiden, U. The feto-placental endothelium in pregnancy pathologies. Wien Med Wochenschr 162, 220-224 (2012). 
47. VanWijk, M. J., Kublickiene, K., Boer, K. \& VanBavel, E. Vascular function in preeclampsia. Cardiovascular Research 47, 38-48 (2000).

48. LeGates, T. A., Kvarta, M. D. \& Thompson, S. M. Sex differences in antidepressant efficacy. Neuropsychopharmacol 44, 140-154 (2019).

49. Myers, S. \& Johns, S. E. Male infants and birth complications are associated with increased incidence of postnatal depression. Social Science \& Medicine 220, 56-64 (2019).

50. Zhang, K. et al. Effect of Toll-like receptor 4 on depressive-like behaviors induced by chronic social defeat stress. Brain and Behavior 10, e01525 (2020).

51. Sternberg, E. M., Trial, J. \& Parker, C. W. Effect of serotonin on murine macrophages: suppression of la expression by serotonin and its reversal by 5-HT2 serotonergic receptor antagonists. J Immunol 137, 276-282 (1986).

52. Sternberg, E. M., Wedner, H. J., Leung, M. K. \& Parker, C. W. Effect of serotonin (5-HT) and other monoamines on murine macrophages: modulation of interferon-gamma induced phagocytosis. $J$ Immunol 138, 4360-4365 (1987).

53. Pedraz-Petrozzi, B., Elyamany, O., Rummel, C. \& Mulert, C. Effects of inflammation on the kynurenine pathway in schizophrenia - a systematic review. Journal of Neuroinflammation 17, 56 (2020).

54. Garrison, A. M. et al. Kynurenine pathway metabolic balance influences microglia activity: targeting kynurenine monooxygenase to dampen neuroinflammation. Psychoneuroendocrinology 94, $1-10(2018)$.

55. Seibenhener, M. L. \& Wooten, M. C. Use of the Open Field Maze to Measure Locomotor and Anxiety-like Behavior in Mice. in J Vis Exp (2015). doi:10.3791/52434.

56. Langmead, B. Aligning short sequencing reads with Bowtie. Current protocols in bioinformatics / editoral board, Andreas D. Baxevanis ... [et al.] CHAPTER, Unit (2010). 
bioRxiv preprint doi: https://doi.org/10.1101/2021.11.12.468408; this version posted November 13,2021 . The copyright holder for this preprint (which was not certified by peer review) is the author/funder, who has granted bioRxiv a license to display the preprint in perpetuity. It is made available under aCC-BY-NC-ND 4.0 International license.

57. Robinson, M. D., McCarthy, D. J. \& Smyth, G. K. edgeR: a Bioconductor package for differential expression analysis of digital gene expression data. Bioinformatics 26, 139-140 (2010).

58. Zhou, Y. et al. Metascape provides a biologist-oriented resource for the analysis of systems-level datasets. Nat Commun 10, 1523 (2019). 